\title{
Progress and Opportunities in EELS and EDS Tomography
}

\author{
Sean M. Collins and Paul A. Midgley \\ Department of Materials Science and Metallurgy, University of Cambridge, \\ 27 Charles Babbage Road, Cambridge CB3 OFS, United Kingdom
}

Email: smc204@cam.ac.uk, pam33@cam.ac.uk

\begin{abstract}
Electron tomography using energy loss and X-ray spectroscopy in the electron microscope continues to develop in rapidly evolving and diverse directions, enabling new insight into the three-dimensional chemistry and physics of nanoscale volumes. Progress has been made recently in improving reconstructions from EELS and EDS signals in electron tomography by applying compressed sensing methods, characterizing new detector technologies in detail, deriving improved models of signal generation, and exploring machine learning approaches to signal processing. These disparate threads can be brought together in a cohesive framework in terms of a model-based approach to analytical electron tomography. Models incorporate information on signal generation and detection as well as prior knowledge of structures in the spectrum image data. Many recent examples illustrate the flexibility of this approach and its feasibility for addressing challenges in non-linear or limited signals in EELS and EDS tomography. Further work in combining multiple imaging and spectroscopy modalities, developing synergistic data acquisition, processing, and reconstruction approaches, and improving the precision of quantitative spectroscopic tomography will expand the frontiers of spatial resolution, dose limits, and maximal information recovery.
\end{abstract}

Keywords: Electron tomography, EELS, EDS, compressed sensing 


\section{Introduction}

ADF-STEM tomography, introduced in 2001, has grown in popularity and is seen now as a routine tool in the study of 3D nanostructure [1-3]. Combining electron tomography with energy loss and X-ray spectroscopy occurred almost concurrently [4-6] but until recently has proven to be far less popular. However, with milestone improvements in energy filters, especially the Gatan Imaging Filter (GIF) [7], in whose development Ondrej Krivanek played such a key role, and, more recently with the development of new X-ray EDS detectors for the TEM, the speed and ease with which analytical signals can be acquired has led to renewed interest among electron tomographers. These advances enable electron tomography to not just address questions of predominantly materials structure but of structure and materials properties simultaneously using EELS and EDS tomography. Elemental composition [8-13], chemical properties such as oxidation state and local bonding environments [14,15], and physical properties such as optical response [16-19] are now measurable in three dimensions at the nanoscale.

Tomography will always seek to determine the relative arrangement of matter in space and, as such, tomography specimens are necessarily "thick" in that they bear some interest due to their three-dimensional structure. In this context, thickness is a relative term, and atomic resolution electron tomography of crystalline specimens just a few nanometers thick is an active ongoing research area in materials microscopy. Importantly, approximations that hold in the limit of thin specimens may not be applicable in general for specimens of interest for tomography. Consequently, linear imaging models underpinning conventional electron tomography may not be valid, particularly in the use of EELS and EDS signals for quantitative measurements. 
Although research in EELS and EDS tomography is developing along many fronts, much of the current focus is on making EELS and EDS tomography quantitative, on applying these methods to new and challenging specimens and signals, and to developing reconstruction methods to extract maximal information from a limited number of, often noisy, spectrum images. Following a brief review and generalization of the methods of electron tomography, current progress in EELS and EDS tomography and future directions are highlighted. Across the board, tools for handling noisy signals from a limited number of spectrum images in a tilt-series are being developed, coupled with the aim of quantitative recovery of structure-property information at the nanoscale. These developments are unified in a model-based approach to analytical electron tomography which will see continued expansion and innovation in future tomography with electron beam imaging spectroscopies.

\section{A model-based framework for analytical electron tomography}

This section outlines some of the key assumptions and equations that underpin tomographic reconstructions as applied to EELS and EDS signals. Traditionally, electron tomography methods can be traced to the concept of the Radon transform, but reconstruction methods in electron tomography today, particularly for EELS and EDS, can be framed within the context of a broader model-based approach, incorporating knowledge of the signal generation process and of the object and information sought in the three-dimensional reconstruction. The relationship between these approaches is outlined to establish clear connections between conventional and emerging tomographic methods and to generalize the variety of state-of-the-art model-based approaches to EELS and EDS tomography. 
The Radon transform of an object is defined over all possible line integrals $L$ along the direction $s$ for an object $f$ :

$$
\mathcal{R}\{f\}=\int_{L} f\left(\mathbf{R}_{\mathbf{0}}^{\prime}(\theta), s\right) d s
$$

with coordinates in the perpendicular plane $\left(\mathbf{R}_{\mathbf{0}}^{\prime}=\left[x^{\prime}, y^{\prime}\right]\right.$ ) determined by the rotation (tilt) angle $\theta$. A single projection $\mathbf{P}_{f}$ can then be written as the Radon transform at a fixed line $L(s=z)$ in a fixed Cartesian coordinate system $\left(\mathbf{R}_{\mathbf{0}}=[x, y], z\right)$ as the line integral along the beam direction $(z)$ :

$$
\mathbf{P}_{f}=\left.\mathcal{R}\{f\}\right|_{s=z}=\int_{-\infty}^{\infty} f\left(\mathbf{R}_{\mathbf{0}}, z\right) d z
$$

Experimentally, the Radon transform is sampled discretely in the form of projections at many tilt angles. Taken together, the measurements acquired during an experiment can be written collectively in terms of a projection operator $\widehat{\mathbf{P}}$ acting on the three-dimensional volume to produce the experimentally recorded projections $\Gamma^{\text {exp }}$

$$
\widehat{\mathbf{P}} f\left(\mathbf{R}_{\mathbf{0}}, z\right)=\Gamma^{\exp } .
$$

This system of equations forms the basis for typical tilt-series tomography reconstruction algorithms such as the algebraic reconstruction technique (ART) and the ubiquitous simultaneous iterative reconstruction technique (SIRT) where a solution for $f\left(\mathbf{R}_{\mathbf{0}}, z\right)$ is found that minimizes the difference between the projected solution and the experimental data. A common way of framing this problem is to minimize the squared differences, equivalent to minimizing the squared $\ell_{2}$-norm:

$$
f\left(\mathbf{R}_{\mathbf{0}}, z\right)=\operatorname{argmin}\left\{\sum_{k}\left(\widehat{\mathbf{P}}_{k} f\left(\mathbf{R}_{\mathbf{0}}, z\right)-\Gamma^{\exp }\right)^{2}\right\}=\operatorname{argmin}\left\{\left\|\widehat{\mathbf{P}} f\left(\mathbf{R}_{\mathbf{0}}, z\right)-\Gamma^{\exp }\right\|_{\ell_{2}}^{2}\right\},
$$

where $k$ denotes the independent beam trajectories contributing to the complete projection operator and norm notation $(\|\cdot\|)$ is used to simplify the equivalent expressions on the right. This expression also highlights several of the limitations intrinsic to tilt-series tomography. Equation (3.) shows that the reconstruction problem is ill-posed (i.e., $f$ may not be a unique solution 
matching the experimental data-set). Additionally, in an experimental realization the Radon transform is highly underdetermined due to the limited number of projections, i.e., there are more unknowns than there are equations. Further, the specific definition of the projection operator in equation (2.) establishes the projection requirement, that the experimental data must be adequately described by the projection operator in the forward model, otherwise approaches to solving equation (4.) will not yield reliable results. This final projection requirement means that the experimental data must be a monotonic function, and preferably a linear function, of the object thickness.

Within this framework there are several possibilities for incorporating additional information about the measurement or the specimen. Such adjustments alter the reconstruction process but critically open up new opportunities for non-linear measurements and enable new algorithms for reconstructing physically significant and quantitative volumes with rich information on the chemistry and physics of the electron-specimen interactions. Together, these changes move electron tomography toward a model-based approach: i.e., that the model can be considered as a description of the signal generation process and also as prior information about the specimen. Each of these models can be incorporated as an amendment to the equations outlined above. Knowledge of the signal generation process leads to replacing the linear projection operator $\widehat{\mathbf{P}}$ with a measurement-specific model. Incorporating details of the EELS signal generation in the excitation of surface plasmons [17], absorption processes in EDS [11], and ADF signal generation [20] fall into this category. Knowledge of the specimen characteristics motivates adding regularization terms to the function to be minimized. The large family of approaches encompassing compressed sensing [21,22] and total variation minimization as well as other regularization approaches [17] and approaches such as discrete tomography 
(DART), the use of Gaussian atomic model priors [23], and methods involving dictionary learning $[24,25]$ or neural network learning [26] procedures form a second category. A third aspect of a model-based approach, model-fitting of the spectral dimension itself splits the problem into several independent reconstructions $(f, g, h, \ldots)$ of separable spectral signatures. Peak fitting, fitting reference spectra, and machine learning techniques for spectral decomposition comprise this third category.

Together, these conceptual approaches generalize the approach to tomographic reconstruction to give:

$$
f\left(\mathbf{R}_{\mathbf{0}}, z\right)=\operatorname{argmin}\left\{\left\|\widehat{\mathbf{\Phi}} f\left(\mathbf{R}_{\mathbf{0}}, z\right)-\Gamma^{e x p}\right\|_{2}^{2}+\lambda\left\|\widehat{\mathbf{\Psi}} f\left(\mathbf{R}_{\mathbf{0}}, z\right)\right\|_{\ell}\right\},
$$

where now the $\widehat{\boldsymbol{\Phi}}$ denotes any operator transforming the object into the experimental signal domain and $\widehat{\boldsymbol{\Psi}}$ denotes any operator transforming the object into a domain where some property of the object can be minimized. The factor $\lambda$ adjusts the weight of the regularization term in the overall solution and $\ell$ highlights that different norms could be applied in this context (e.g., selection of the $\ell_{1}$-norm as used in compressed sensing promotes the sparsest solution in the transform domain defined by $\widehat{\boldsymbol{\Psi}}$. A signal is said to be $k$-sparse if it contains $k$ non-zero values.).

This categorization is not necessarily rigid. Refinement of tilt-series image alignments [23] during the reconstruction process makes use of knowledge of the specimen, as it relies on using the structure of the specimen for iterative corrections to the alignment, but the procedure could be written as modifying the forward-model term $\widehat{\mathbf{\Phi}} f\left(\mathbf{R}_{\mathbf{0}}, z\right)$. However, the general distinction of development of forward models of signal generation $(\widehat{\boldsymbol{\Phi}})$ and specimen-structure models $(\widehat{\Psi})$ serves to emphasize these two often complementary strands emerging in electron tomography today, particularly as tomography methods are increasingly applied to analytical 
signals in the electron microscope where model-based approaches become imperative for quantitative chemical and structural materials characterization.

\section{Recent progress in EELS and EDS tomography}

Recent developments of the GIF and large-area EDS detectors in both speed and efficiency have made EELS and EDS tomography possible, but both intrinsic problems and technical challenges associated with spectroscopy in the TEM present difficulties for tomography using EELS and EDS signals. Due to the cross-sections for inelastic electron scattering and X-ray generation [27], the signal-to-noise ratio for a given electron dose at the specimen is significantly lower in EELS and EDS than in ADF-STEM. To compensate, higher

electron doses are often used experimentally either by increasing the beam current, increasing the dwell time (or exposure time in EFTEM), or some combination of both. This higher electron dose in turn creates several significant problems in electron tomography. The time to acquire the data is long, placing more stringent requirements on both chemical and mechanical stability of the specimen, or the specimen may change during the acquisition introducing artefacts. These artefacts appear as blurring or streaking in the reconstructed volume due to inconsistencies in the tilt-series data that break the projection requirement for a monotonic signal response (see also discussion in Ref. [28]). The data is often still noisier for EELS and EDS than that acquired in ADF-STEM, and often spectrum images are acquired at fewer tilt angles due to specimen stability and time constraints. Moreover, for EELS and EDS the spectrum imaging conditions for meeting the projection requirement are rigorously satisfied only within a narrow range of specimen thicknesses (and the projected specimen thickness may change significantly during a tilt-series acquisition), or the projection requirement is valid only for certain types of 
spectroscopic signals (e.g., low-loss EELS signals such as surface plasmon excitations do not in general satisfy the projection requirement). Coherent elastic scattering effects, including electron channeling in atomic resolution imaging and dynamical scattering in strongly diffracting samples, modify the inelastic scattering signals recorded in analytical tilt-series experiments, introducing further deviations from linear projection-based tomography. Additionally, technical challenges appear in that data-sets are increasingly large in terms of computational memory requirements for processing, reconstruction, and visualization, and the information content is more challenging to interpret due to its multi-dimensionality.

Recent progress in electron tomography using EELS and EDS signals has provided a number of different strategies for addressing these obstacles to quantitative analytical electron tomography. While these developments have so far often been applied as tailor-made solutions for particular materials applications or for technique demonstrations, several common themes suggest a generalized suite of approaches to developing quantitative tomography and for applying these techniques to a wider set of materials.

\subsection{Compressed sensing tomography}

Compressed sensing and related TV minimization approaches have offered substantial advancements to electron tomography using ADF-STEM, particularly in enabling the acquisition of fewer projections for robust reconstructions. These reconstruction techniques find natural application in EELS and EDS tomography as specimen and signal-to-noise requirements often result in spectrum images acquired at fewer tilts and correspondingly larger tilt angle increments. As alluded to in Sec. 2, compressed sensing makes use of the inherent sparsity of many objects in one or more transform domains. For example, some objects may inherently consist of only a 
few non-zero voxels and are therefore sparse in the image domain (identity matrix as the transform operator). Many objects are approximately piece-wise smooth, and are described well by only a few non-zero voxels in the gradient domain (TV minimization). By incorporating this prior knowledge, compressed sensing algorithms identify solutions consistent with the experimental data that also exhibit these characteristic properties of common specimen structures. Compressed sensing electron tomography in this form has been applied to a number of problems in EELS and EDS tomography, including qualitative EELS tomography of surface plasmons in a silver nanocube [16], combined EELS and EDS tomography of an Al-Si alloy [8], and three-dimensional EELS oxidation state mapping in iron oxide nanoparticles [15], see Fig. 1.

(a)

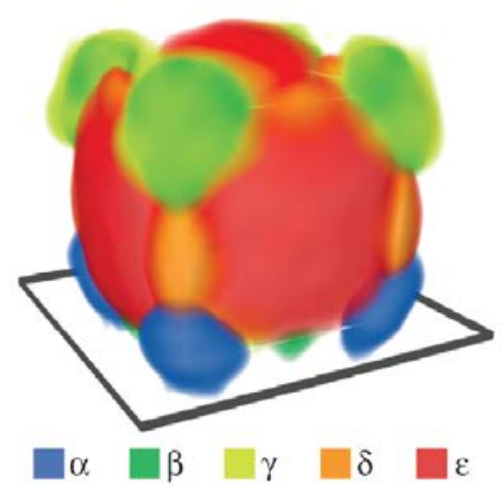

(b)

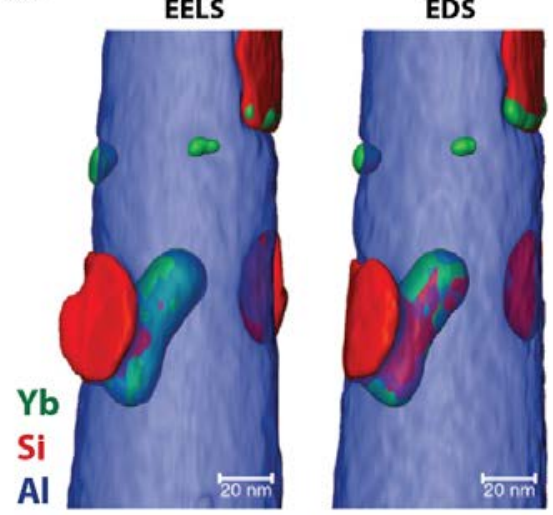

(c)

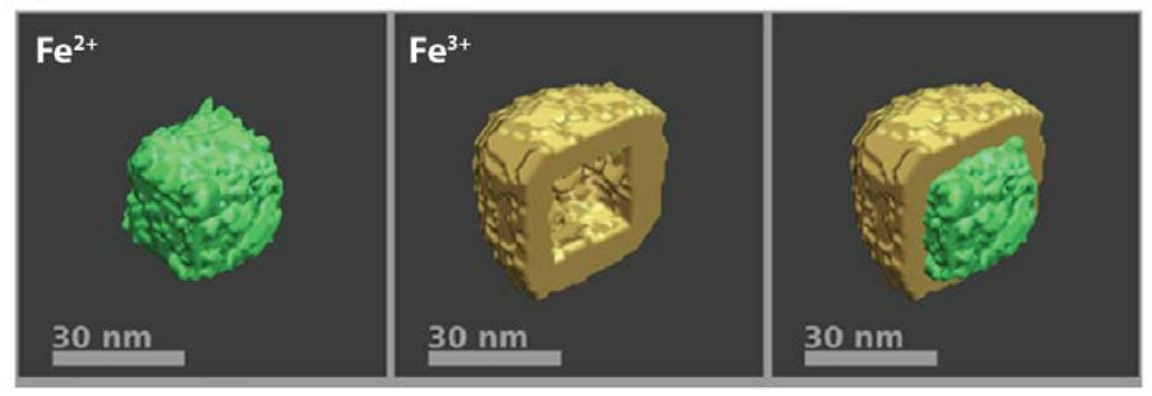

Fig. 1. (a) Compressed sensing electron tomography (CS-ET) reconstruction of five surface plasmon modes of a silver nanocube using a wavelet transform for the sparsity domain [16]. (b) 3D total variation (TV) voxel tomography reconstructions of EELS and EDS signals in an Al-Si alloy. Adapted from Ref. [8] with permission of The Royal Society of Chemistry. (c) CS-ET reconstruction of the EELS signatures of $\mathrm{Fe}^{2+}$ and $\mathrm{Fe}^{3+}$ using independent component analysis (ICA) maps as tilt-series input. Adapted with permission from Ref. [15]. Copyright 2016 American Chemical Society. 
The application of compressed sensing to analytical signals must be done with care. For example, in the case of EELS tomography of surface plasmons the transform domain for the surface plasmon EELS signal was selected as a wavelet transform domain to allow for the continuous characteristics of the slowly decaying surface plasmon signal extending into vacuum from the nanoparticle surface, a signal that is not particularly sparse in the image or TV domains [16]. The question of the appropriate transform domain has been offered as a potential limitation in other applications of compressed sensing to electron tomography, such as in the case of biological structures whose images may not be sufficiently sparse in many of the conventionally applied transform domains [29].

A promising development in the field of compressed sensing that holds substantial promise for analytical tomography is the extension of the sparsifying operator to the third dimension. In applications of spectroscopic tomography where each spectral channel is used for a reconstruction $[14,30]$ parallelization of reconstructions has often been prioritized using SIRT algorithms. These parallelized reconstructions rely on 'slice-by-slice' tomography where each row perpendicular to the tilt-axis in the projection image stack is handled as an independent reconstruction. However, if parallelization is sacrificed in favor of sparsity models that incorporate the sparsity of the fully three-dimensional object, arguably the true sparsity of the object, then reconstructions taking into account the information in the dimension running parallel to the tilt axis will be promoted, improving the overall self-consistency of the reconstructed volume. Such a three-dimensional TV approach has been applied for combined EELS and EDS tomography [8] and has also shown progress in ADF-STEM for improved reconstructions from data suffering artefacts due to diffraction contrast and wide tilt angle increments [28]. This 
approach, particularly in combination with signal dimension reduction approaches such as machine learning techniques or peak fitting, offers a complementary strategy to massively parallel independent reconstructions which may otherwise be noise-limited. Other potential developments of interest to analytical electron tomography have arisen in the combination of TV minimization and DART [31] which may enable high quality reconstructions of materials consisting of a few discrete chemical phases. While not necessarily always the best choice for a particular tomography problem, compressed sensing techniques hold significant promise for improving analytical electron tomography using limited projections.

\subsection{EELS tomography}

Electron tomography with EELS signals was originally developed in the context of energy-filtered TEM (EFTEM) for tomography [4,5]. Recent developments in EELS tomography have emerged in two principle directions: (1) tomography using low energy signals such as those arising from surface plasmon excitations in low-loss EELS of metal nanoparticles and in cathodoluminescence (CL) spectroscopy and (2) tomography using core-loss EELS with increased focus on the near edge fine structure for valency and chemical phase mapping available at moderate to high spectral energy resolution. The increased experimental energy resolution at low- and high-energy losses can be directly linked to improvements in the GIF and related spectrometers, both in energy resolution and acquisition speed, as well as a drive for high resolution monochromators for the incident electron probe. Efforts were previously largely focused on EFTEM tomography using low-energy losses at bulk plasmon energies, particularly as a means of achieving chemical contrast with short exposure times [32-35]. And while a variety of low-loss signals have been explored through EELS studies in general, the low-loss 
spectral range is rich with opportunities for further tomographic EELS studies. Core-loss EELS tomography is a natural extension of elemental and chemical phase mapping in EFTEM, but presents distinct challenges for data pre-processing and reconstruction. Core-loss EELS tomography is a natural extension of elemental mapping in EFTEM, but presents distinct challenges for data pre-processing and reconstruction. Tomography using low energy signals like surface plasmon EELS and CL raises entirely new questions about meeting the projection requirement for tomography.

Initial work in EELS tomography of surface plasmons successfully applied a projectionbased approach to reconstruction of the major surface plasmon modes of a silver nanocube [16]. The integral determining the EELS signal for a given trajectory includes a complex exponential factor due to the frequency-domain properties of the incident electron beam. This complex exponential approaches unity for small arguments, approximating a line integral projection. However, this projection-based method relies on a set of approximations of the surface plasmon EELS signal which, while suitably satisfied in the case of the nanocube, are unlikely to hold generally. A similar linear projection-based reconstruction was subsequently applied using a multi-particle approach rather than a tilt-series protocol for CL tomography of gold plasmonic crescents [19], see Fig. 2(a). A further projection-based approach has also been reported on dimer particle structures [18]. For quantitative EELS excitations of surface plasmons, the nonlinear details of the signal generation mechanism should be taken into account. Moreover, in terms of a useful signal for understanding optical properties, the EELS signal does not directly record the essential information for optical characterization. In the loss function formalism, EELS signals are related to the complex dielectric constant of a material, but such approaches require additional processing steps such as carefully considered Kramers-Kronig analysis and 
complete modelling of surface and relativistic effects [36]. Accessing the underlying information about the specimen that is of particular relevance to understanding the property of interest needs to be the aim of the tomography experiment.

Using theoretical developments for modelling the EELS signal of surface plasmons, Hörl et al. proposed two alternative approaches to EELS tomography of surface plasmons $[37,38]$. Both of these approaches rely on a model-based reconstruction method, incorporating detailed, and critically non-linear, forward models of signal generation for comparison with experimental data. In the first approach, the forward model consists of an eigenmode description of the surface plasmon EELS signal which reduces the forward model to an integral over surface charges distributed across the two-dimensional boundary of the particle [37] in contrast to line integrals through the particle volume. Iterative equation solvers can then be applied to refine the surface charges. An alternative method with fewer approximations limiting applicable particle sizes was proposed using a function basis set to further model the response of the particle, likewise making use of iterative refinements to match experimental data [38], see Fig. 2(b). Similar theoretical developments in CL of surface plasmons suggest these tomographic approaches could be extended to CL [39].

The first approach derived from an eigenmode decomposition of the EELS signal has been realized in the experimental case of a silver right bipyramid [17], see Fig. 2(c). In the case of the silver right bipyramid reconstructions, surface charges were reconstructed giving a quantitative signal recovered during tomographic reconstruction for simulating the optical response characteristics of the bipyramid particle for any plane wave or other incident electromagnetic excitation. An additional regularization term incorporating prior knowledge of the distribution of surface charge magnitudes in the bipyramid surface plasmons was also shown 
to improve reconstructions in the context of noisy experimental signals. These developments illustrate progress in the use of forward-models of signal generation and the incorporation of prior knowledge of properties of the signal undergoing reconstruction.

(a)
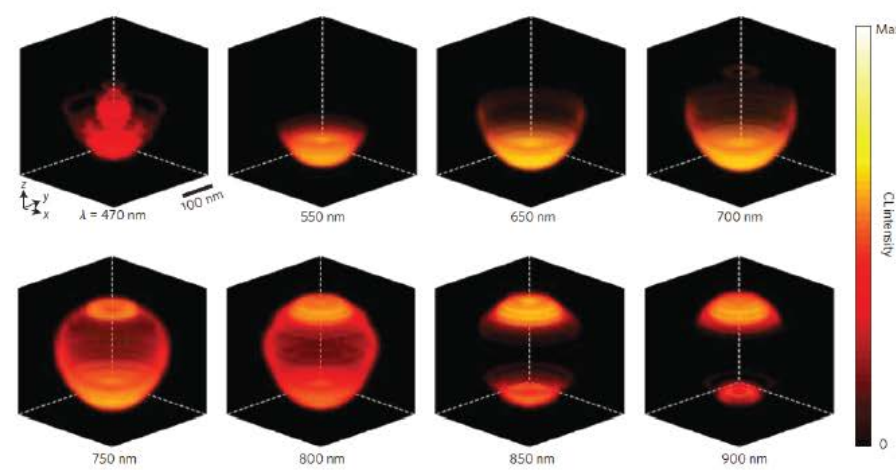

(b)

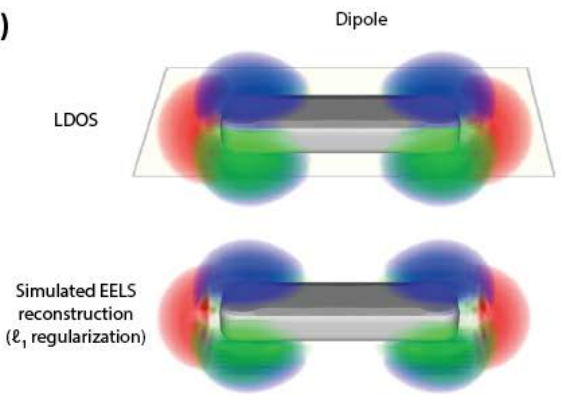

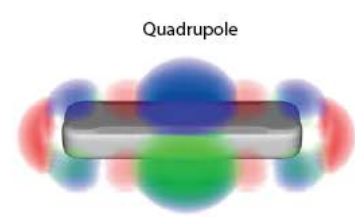

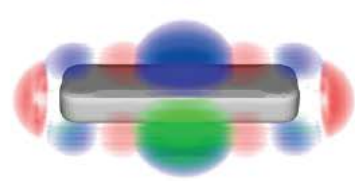

(c)
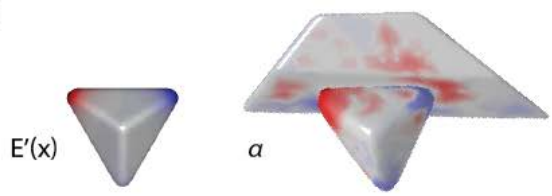

$E^{\prime}(y)$
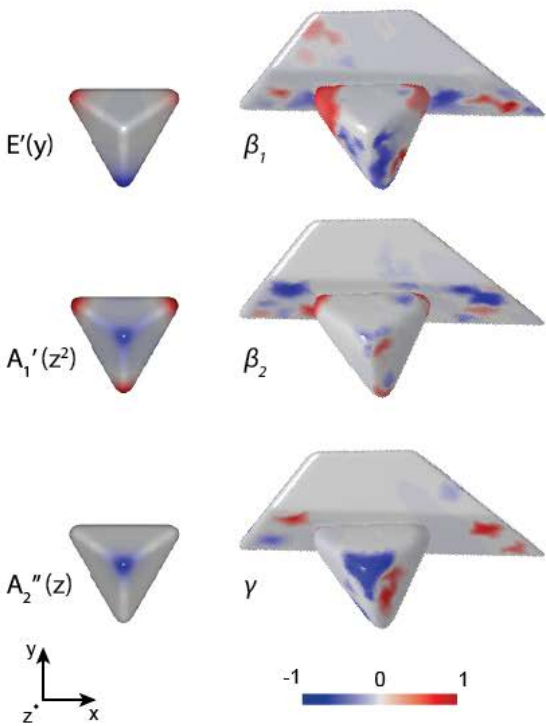

Fig. 2. (a) Cathodoluminescence tomography of surface plasmon modes of gold nanocrescents. Adapted by permission from Macmillan Publishers Ltd: Nature Nanotechnology Ref. [19], copyright 2016. (b) A proposed EELS reconstruction approach for tomography of the photonic LDOS [38]. (c) Simulated and experimentally reconstructed surface plasmon eigenmodes of a silver right bipyramid using combined CS ADF-STEM tomography and a regularized non-linear EELS reconstruction algorithm [17].

The issues raised in surface plasmon EELS tomography and some of the means of addressing them also suggest further opportunities for development of other low-loss EELS signals for tomography. Three-dimensional measurements of band gaps and interband transitions, excitons, and other low-energy signals will provide new insight into the physical properties of specimens. Many of these low-loss signals are emerging as areas of active exploration in two-dimensional analyses by EELS with the use of new electron beam 
monochromators [40,41]. Further developments in EELS at infrared energies may also offer new signals, though issues of signal localization will be critical for examining any application to tomography [42]. Complementary research in CL of semiconductors [43] and single-photon emitters [44] have also broadened the frontiers of spectroscopy in the TEM. Each of these applications may require consideration of the information sought for recovery in a tomographic reconstruction and of the forward models needed to replicate the signal generation from the physical properties of interest, but these measurements offer a wealth of new opportunities for quantitative measurement of physical properties of nanoscale volumes.

Core-loss EELS tomography has also seen recent progress in revealing detailed chemical information from a variety of materials from catalyst materials [13-15] to organic polymer blends [30]. Several new thrusts in EELS tomography research have been based around new reconstruction algorithms, including massively parallelized GPU-accelerated reconstructions [14] and three-dimensional TV minimization strategies [8], as well as in the advancement of data pre-processing strategies. Data pre-processing methods for noise reduction and dimensionality reduction of the spectrum image tilt-series data, while preserving the spectral features of interest, is a common feature in low-loss and core-loss EELS. It is possible to perform 'voxelspectroscopy' $[8,14]$ where the EELS signal is reconstructed in three spatial dimensions at every energy channel, but this approach requires sufficiently high-quality signal at every energy channel. Noisy spectral data may introduce artefacts in tomographic reconstructions which complicate post-processing. At the opposite extreme, using elemental maps based on background-subtracted ionization edge intensities, the STEM-EELS analog of EFTEM tomography, very little information other than the elemental composition can be extracted. Peak fitting and machine learning approaches offer a middle-ground that has the potential to embed 
chemical phase analysis and chemical composition quantification simultaneously. In specimens that contain a variety of phases with shared elemental constituents, the information of interest is in fact the composition and properties of separate phases. By grouping EELS signals using a model-fitting technique like peak fitting, fitting of reference spectra, or machine learning, the tomographic reconstruction is simplified into a small number of reconstructions matching the number of chemical phases rather than the number of energy channels. Moreover, the desired information of chemical speciation is contained within the reconstruction from data with noise substantially lower than the noise present in the originally acquired data.

Model-based approaches in data pre-processing can take a number of different forms. Perhaps in its simplest incarnation, principal component analysis (PCA) can be used for noisereduction prior to elemental map-based reconstructions [13]. Alternatively, any number of machine learning decomposition strategies can be applied to EELS data to generate component maps, plots of the intensity of particular spectral signal components at each pixel in the image. PCA has been used to directly extract chemical phase-specific information in organic solar cell mixtures to determine suitable energy-windows in EFTEM tomography [45]. Non-negative matrix factorization (NMF) was applied on the entire tilt-series spectrum image data-set in surface plasmon EELS tomography experiments to extract tilt-series maps of separable surface plasmon modes [16,17]. Independent component analysis (ICA) of core-loss EELS has also been applied to extract component maps corresponding to different valency states in iron oxide nanoparticles for tomography [15]. These machine learning algorithms extract useful chemical information with reduced noise as input for tomography. One of their shortcomings, however, is the quantitative physical interpretation of spatial map intensities. In principle, peak fitting or fitting of reference spectra presents an alternative strategy, but multi-dimensional peak fitting is 
not a trivial task in the context of experimental noise and the many data-points in a spectrum image tilt-series. Peak fitting approaches also require a great deal of prior knowledge of the chemical composition of the specimen. Further progress in validating the physical interpretation of machine learning results and the development of peak fitting strategies for such data-sets will foster wider application of these methods.

In the core-loss EELS tomography examples presented here, the forward model for signal generation has generally been a linear projection model. The use of non-linear models in lowloss EELS suggests similar approaches might be used in core-loss EELS tomography as well. The motivation for using non-linear forward models lies in the fact that EELS through volumes is not strictly a projection, particularly at core-loss energies. Inelastic scattering consists of the single scattering distribution as well as plural scattering contributions which may be expressed as a convolution with the low-loss EELS signal. EELS tomography necessarily examines specimens with information of interest located in the volume of the specimen and is of primary interest in specimens with mixed chemical composition. Consequently, thickness-dependent plural scattering must be accounted for in generalized, quantitative EELS tomography. One approach may lie in recovering the single-scattering distribution during data pre-processing through deconvolution steps, possibly making use of simultaneously recorded low-loss and core-loss spectra (“dualEELS”). Alternatively, forward models that account for plural scattering effects may make better use of the total data-set during the reconstruction. The success of machine learning techniques suggests that there are substantial benefits to be gained from the redundancy of information present in spectrum image data-sets, particularly in cases of tilt-series data-sets. Model-based pre-processing and forward models that incorporate plural scattering effects offer new approaches that take full advantage of the available EELS data. As the trend toward using 
fewer projections continues, it will become increasingly imperative that all available signals are used as efficiently as possible in EELS tomography.

\subsection{EDS tomography}

Early work in EDS tomography identified severe limitations in tilt-range and signal-tonoise with $\mathrm{Si}(\mathrm{Li})$ detectors [46]. More recently, the prospects for EDS tomography have been revolutionized by the development of large-area silicon drift detectors (SDDs) for STEM-EDS. Current-generation SDD systems typically place multiple detectors in the TEM pole-piece gap for much higher $\mathrm{X}$-ray collection rates than previously achievable with $\mathrm{Si}(\mathrm{Li})$ detectors and an overall substantial increase in solid angle subtended by the multiple detectors. Qualitative EDS tomography results have been reported with these systems including work on mixed-metal nanoparticles $[9,10]$ and Ni-based superalloys [12]. However, detector shadowing $[47,48]$ and Xray absorption $[11,49,50]$ effects have emerged as two key challenges requiring various corrections for quantitative EDS tomography. Shadowing can in principle be avoided completely by using a needle-shaped specimen as prepared by FIB milling [12], see Fig. 3(a), but such preparation is not a viable approach for all specimens.

One approach has been to measure as much as possible about the particular experimental configuration [47], akin to the requirements for quantitative STEM aimed at assessing the significance of the many parameters contributing to an atomic-resolution ADF image. In this approach to EDS, detailed analysis of the holder and detector geometry can be used to assess shadowing as a function of tilt angle. However, as in quantitative STEM imaging, models with only a few parameters that incorporate the most significant contributing factors are attractive for widespread application of corrections for detector shadowing [48] or X-ray absorption [49]. 
Absorption corrections have been proposed within both the Cliff-Lorimer [50] and the $\zeta$ factor [49] approaches. In fact, absorption effects appear in both models in the same form [11]. It has been proposed that the $\zeta$-factor approach enables the incorporation of both shadowing and absorption effects for a particular experimental system [49] when the $\zeta$-factors are determined by for the same system using single element reference specimens measured by ADF tomography [51]. In this approach, absorption corrections are applied to individual EDS maps at each tilt angle. Consequently, ADF data must be used to supplement the EDS maps to incorporate thickness information at each tilt [49]. This method presents an efficient multi-modal approach to using available EDS and ADF-STEM data. However, these absorption corrections cannot account for the path-dependent absorption effects from within the volume unless the chemical phase distribution is determined already, e.g. from ADF-STEM tomography. A more comprehensive approach has been developed to iteratively adjust absorption corrections using the intermediate tomogram to correct for the path-dependent absorption of X-rays reaching a particular detector [11], see Fig. 3(b). With more constrained prior information on the structure of the specimen, it is even possible to use a highly limited number of tilt angles and fit EDS data, including measured $\zeta$-factors, to a structural-chemical model [52], see Fig. 3(c). There is almost certainly always a trade-off in these approaches between constraining the reconstruction with a rigid model, allowing for the use of very few tilt angles, and reducing the prior information but consequently requiring more experimental data to perform the reconstruction.

In any case, it is clear that quantitative EDS tomography will require corrections for absorption and detector shadowing effects either in data pre-processing or during the tomographic reconstruction procedure. Additional effects such as X-ray fluorescence may also become more prominent in thicker specimens. Machine learning techniques will likely continue 
to play a significant role in EDS data handling, as in EELS tomography, and have already shown substantial results in two- [53] and three-dimensional [12] EDS of complex materials.

(a)

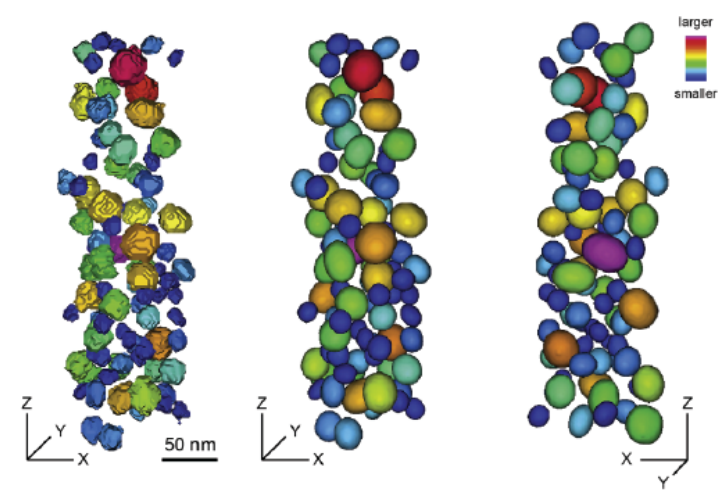

(b)

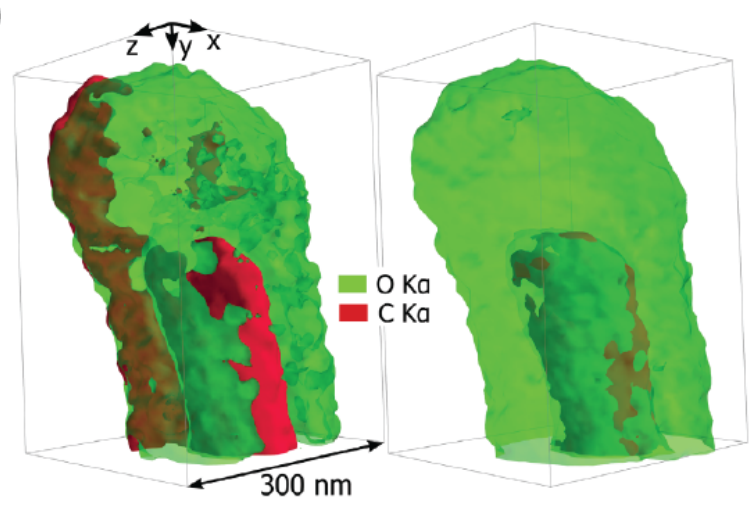

(c)

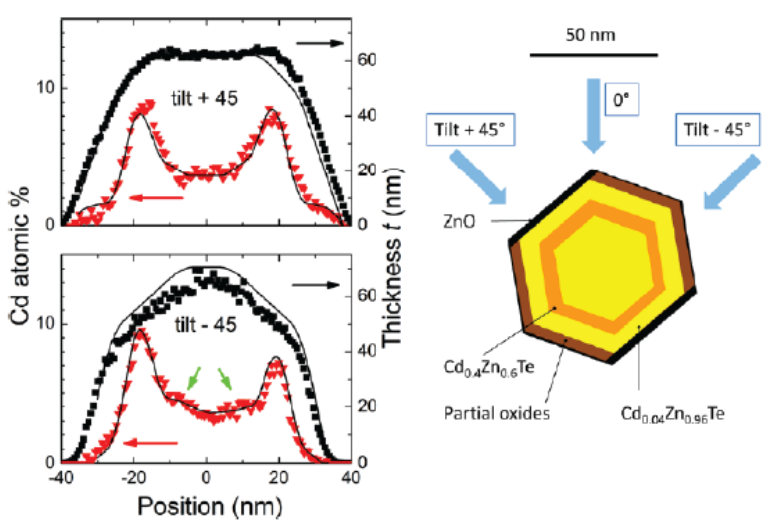

Fig. 3. (a) EDS tomography of the $\gamma^{\prime}$ strengthening phase of a Ni-based superalloy using ICA decomposition techniques for pre-processing and ellipsoid fitting for post-processing [12]. (b) EDS tomography of carbon and oxygen in a multi-element core-shell nanowire using (left) a conventional reconstruction approach and (right) an iterative model-based approach to absorption correction [11]. (c) A structural model-based approach to 3D EDS quantification of core-shell semiconductor nanowires from limited projection data using $\zeta$-factor absorption correction methods. Adapted with permission from Ref. [52]. Copyright 2016 American Chemical Society. 


\section{Challenges and future prospects}

The goal of EELS and EDS tomography ultimately lies in achieving precise, quantitative measurements of chemical and physical properties of nanoscale volumes. Tomography presents particular opportunities as well as obstacles to quantitative spectral analysis. Model-based tomographic approaches can enable the extraction of new or improved spectroscopic measurements in comparison to two-dimensional measurements alone: surface charge reconstructions of plasmonic excitations [17] and $\zeta$-factor determination from ADF tomography [51] both use tomography and analytical signals to advance spectral analysis questions in addition to three-dimensional signal recovery. However, EELS and EDS tomography results for quantification are also plagued by artefacts in reconstructed volumes. Grey-levels with significant intensity outside of the specimen structure may appear in reconstructed volumes due to the limited number of tilt-angles, mis-alignments in the tilt-series, changes in the specimen over the course of the data acquisition, or noise in the experimental data. These non-physical grey-levels still contribute to the match between experimental data and re-projections of the volume, but are not accounted for in individual 'voxel spectra' or voxel composition data in elemental reconstructions. In these cases, there will be a certain amount of information that has been smeared throughout the reconstruction volume, resulting in 'missing' intensity in the subvolume of the reconstructed object relative to the re-projected tilt-series (and the corresponding original data). Spectral model-fitting may avoid these issues because any quantification can be achieved separately on the spectral components of the model and the reconstruction operates only on the spatial distribution of a particular component. 
Model-based approaches present their own challenges, however. Key issues include (a) avoiding designs that determine too many model parameters from insufficient data, (b) determining the optimal regularization parameters, number of model components, and number of iterations, (c) identifying appropriate initializations for iterative algorithms, and (d) finding the best match for sampling and sensing schemes during an experiment. Validation and testing with phantom data-sets may provide some guide to these questions, but in some cases additional theoretical developments in compressed sensing and related sampling theories will be required to provide a robust foundation for experimental choices.

Computational requirements for increasingly complex models for signal generation and for increasingly large data-sets, in terms of spectral channel depth and image size at each tilt angle, are likewise growing. New approaches such as three-dimensional regularization in compressed sensing also require larger amounts of memory for each reconstruction. While hardware needs may be surmountable, efficient software will become an increasingly vital part of EELS and EDS tomography. Efficient algorithm implementations that are portable across platforms and accessible to users worldwide will likely reap the most benefit. Open source projects like the Astra Toolbox [54] for tomography and Hyperspy [55] for EELS and EDS processing are key examples advancing this research area.

Experimentally, advances in instrumentation will continue to have a crucial role in moving the frontiers of EELS and EDS tomography. Electron beam monochromators and fastacquisition GIF and SDD technologies for EELS and EDS, respectively, have substantially enabled progress. New monochromator designs by Nion are opening further possibilities in vibrational EELS and core-loss spectroscopy at high energy and spatial resolution. The limited flexibility of acquisition software and the underlying hardware platforms, however, remains a 
significant obstacle for exploring new experimental possibilities for analytical tomography. Free control of beam scanning and blanking, independent read-out of multiple EDS detectors, and moving from CCDs to low-noise direct electron detectors, among other developments, will enable the next set of advances in this burgeoning field.

Analytical electron tomography will also grow with application of these techniques to new materials and new signals. Low-energy signals below $50 \mathrm{eV}$ in EELS offer a wealth of information on chemical and physical properties, but are often used only to remove plural scattering from core-loss spectra. These EELS signals are in many cases sufficiently intense to acquire at or near ADF-STEM imaging rates, offering new prospects for tomographic chemical analysis. Additional signals like CL spectroscopy as well as the use of spectroscopic signals in tandem with scanning diffraction data will offer new materials insights into chemical, optical, and crystallographic microstructure. Comparative measurements with other techniques like atom probe tomography merit further study for improved quantification and cross-validation of microanalysis results. Radiation-sensitive materials, conventionally deemed inaccessible to the high beam currents or long exposure times required for analytical tomography, are becoming accessible through careful experimental design and dose-rationing enabled by model-based approaches. In addition to compressed sensing and similar approaches to tomography which may reduce the total dose required for a satisfactory reconstruction by reducing the number of projections, machine learning approaches which reduce the required dose rate by enabling the use of signals with higher noise levels or machine learning approaches which enable reconstruction of time-dependent sample changes throughout a tilt-series due to electron beaminduced damage promise to substantially reduce dose requirements for analytical electron tomography. 


\section{Summary and Outlook}

Recent progress in EELS and EDS tomography has advanced three-dimensional chemical imaging at high spatial resolution in a variety of exciting new directions. More than simply

elemental mapping, EELS tomography is now demonstrating the possibility of mapping physical and chemical behaviour through nanoscale mapping of optical properties to valence states with nanometer resolution. EDS tomography has also progressed to the point where quantitative three-dimensional elemental X-ray mapping is possible in the STEM. These advances open new questions for developing improved models, models for signal generation, models for incorporating prior knowledge for physical reconstructions, and models for spectral processing. Theoretical and algorithmic developments alongside further progress in spectrometer and microscope technologies will pave the way for making possible new, precise measurements of nanoscale three-dimensional chemistry and physics.

\section{Acknowledgements}

SMC acknowledges support from the EPSRC Cambridge NanoDTC, EP/G037221/1, and Trinity College, Cambridge. SMC and PAM also acknowledge support from the European Research Council under the European Union’s Seventh Framework Program (No. FP7/20072013)/ERC Grant Agreement No. 291522-3DIMAGE.

\section{References}

[1] P. Ercius, O. Alaidi, M.J. Rames, G. Ren, Electron Tomography: A Three-Dimensional Analytic Tool for Hard and Soft Materials Research, Adv. Mater. 27 (2015) 5638-5663. doi:10.1002/adma.201501015. 
[2] Z. Saghi, P.A. Midgley, Electron Tomography in the (S)TEM: From Nanoscale Morphological Analysis to 3D Atomic Imaging, Annu. Rev. Mater. Res. 42 (2012) 59-79. doi:10.1146/annurev-matsci-070511-155019.

[3] G. Van Tendeloo, S. Bals, S. Van Aert, J. Verbeeck, D. Van Dyck, Advanced Electron Microscopy for Advanced Materials, Adv. Mater. 24 (2012) 5655-5675. doi:10.1002/adma.201202107.

[4] M. Weyland, P.A. Midgley, 3D-EFTEM: Tomographic reconstruction from tilt series of energy loss images, in: Conf. Ser. - Inst. Phys., Philadelphia; Institute of Physics; 1999, 2001: pp. 239-242.

[5] G. Möbus, B.J. Inkson, Three-dimensional reconstruction of buried nanoparticles by element-sensitive tomography based on inelastically scattered electrons, Appl. Phys. Lett. 79 (2001) 1369-1371. doi:10.1063/1.1400080.

[6] P.A. Midgley, M. Weyland, J.M. Thomas, B.F.G. Johnson, Z-Contrast tomography: a technique inthree-dimensional nanostructural analysis based on Rutherfordscattering, Chem. Commun. (2001) 907-908. doi:10.1039/B101819C.

[7] O.L. Krivanek, A.J. Gubbens, N. Dellby, C.E. Meyer, Design and first applications of a post-column imaging filter, Microsc. Microanal. Microstruct. 3 (1992) 187-199. doi:10.1051/mmm:0199200302-3018700.

[8] G. Haberfehlner, A. Orthacker, M. Albu, J. Li, G. Kothleitner, Nanoscale voxel spectroscopy by simultaneous EELS and EDS tomography, Nanoscale. 6 (2014) 1456314569. doi:10.1039/C4NR04553J.

[9] B. Goris, L. Polavarapu, S. Bals, G. Van Tendeloo, L.M. Liz-Marzán, Monitoring Galvanic Replacement Through Three-Dimensional Morphological and Chemical Mapping, Nano Lett. (2014). doi:10.1021/nl500593j.

[10] T.J.A. Slater, A. Macedo, S.L.M. Schroeder, M.G. Burke, P. O’Brien, P.H.C. Camargo, S.J. Haigh, Correlating Catalytic Activity of Ag-Au Nanoparticles with 3D Compositional Variations, Nano Lett. (2014). doi:10.1021/nl4047448.

[11] P. Burdet, Z. Saghi, A.N. Filippin, A. Borrás, P.A. Midgley, A novel 3D absorption correction method for quantitative EDX-STEM tomography, Ultramicroscopy. (n.d.). doi:10.1016/j.ultramic.2015.09.012.

[12] D. Rossouw, R. Krakow, Z. Saghi, C.S.M. Yeoh, P. Burdet, R.K. Leary, F. de la Peña, C. Ducati, C.M.F. Rae, P.A. Midgley, Blind source separation aided characterization of the $\gamma^{\prime}$ strengthening phase in an advanced nickel-based superalloy by spectroscopic 4D electron microscopy, Acta Mater. 107 (2016) 229-238. doi:10.1016/j.actamat.2016.01.042.

[13] L. Yedra, A. Eljarrat, R. Arenal, L. López-Conesa, E. Pellicer, A. López-Ortega, M. Estrader, J. Sort, M.D. Baró, S. Estradé, F. Peiró, Electron energy-loss spectroscopic tomography of $\mathrm{FexCo}(3-\mathrm{x}) \mathrm{O} 4$ impregnated $\mathrm{Co} 3 \mathrm{O} 4$ mesoporous particles: unraveling the chemical information in three dimensions, Analyst. (2016). doi:10.1039/C6AN00562D.

[14] B. Goris, S. Turner, S. Bals, G. Van Tendeloo, Three-Dimensional Valency Mapping in Ceria Nanocrystals, ACS Nano. (2014). doi:10.1021/nn5047053.

[15] P. Torruella, R. Arenal, F. de la Peña, Z. Saghi, L. Yedra, A. Eljarrat, L. López-Conesa, M. Estrader, A. López-Ortega, G. Salazar-Alvarez, J. Nogués, C. Ducati, P.A. Midgley, F. Peiró, S. Estradé, 3D Visualization of the Iron Oxidation State in FeO/Fe3O4 Core-Shell Nanocubes from Electron Energy Loss Tomography, Nano Lett. 16 (2016) 5068-5073. doi:10.1021/acs.nanolett.6b01922. 
[16] O. Nicoletti, F. de la Peña, R.K. Leary, D.J. Holland, C. Ducati, P.A. Midgley, Threedimensional imaging of localized surface plasmon resonances of metal nanoparticles, Nature. 502 (2013) 80-84. doi:10.1038/nature12469.

[17] S.M. Collins, E. Ringe, M. Duchamp, Z. Saghi, R.E. Dunin-Borkowski, P.A. Midgley, Eigenmode Tomography of Surface Charge Oscillations of Plasmonic Nanoparticles by Electron Energy Loss Spectroscopy, ACS Photonics. 2 (2015) 1628-1635. doi:10.1021/acsphotonics.5b00421.

[18] G. Haberfehlner, A. Trügler, F.P. Schmidt, A. Hörl, F. Hofer, U. Hohenester, G. Kothleitner, Correlated 3D Nanoscale Mapping and Simulation of Coupled Plasmonic Nanoparticles, Nano Lett. 15 (2015) 7726-7730. doi:10.1021/acs.nanolett.5b03780.

[19] A.C. Atre, B.J.M. Brenny, T. Coenen, A. García-Etxarri, A. Polman, J.A. Dionne, Nanoscale optical tomography with cathodoluminescence spectroscopy, Nat. Nanotechnol. 10 (2015) 429-436. doi:10.1038/nnano.2015.39.

[20] S.V. Venkatakrishnan, L.F. Drummy, M.A. Jackson, M.D. Graef, J. Simmons, C.A. Bouman, A Model Based Iterative Reconstruction Algorithm For High Angle Annular Dark Field-Scanning Transmission Electron Microscope (HAADF-STEM) Tomography, IEEE Trans. Image Process. 22 (2013) 4532-4544. doi:10.1109/TIP.2013.2277784.

[21] Z. Saghi, D.J. Holland, R. Leary, A. Falqui, G. Bertoni, A.J. Sederman, L.F. Gladden, P.A. Midgley, Three-Dimensional Morphology of Iron Oxide Nanoparticles with Reactive Concave Surfaces. A Compressed Sensing-Electron Tomography (CS-ET) Approach, Nano Lett. 11 (2011) 4666-4673. doi:10.1021/nl202253a.

[22] R. Leary, Z. Saghi, P.A. Midgley, D.J. Holland, Compressed sensing electron tomography, Ultramicroscopy. 131 (2013) 70-91. doi:10.1016/j.ultramic.2013.03.019.

[23] B. Goris, J. De Beenhouwer, A. De Backer, D. Zanaga, K.J. Batenburg, A. SánchezIglesias, L.M. Liz-Marzán, S. Van Aert, S. Bals, J. Sijbers, G. Van Tendeloo, Measuring Lattice Strain in Three Dimensions through Electron Microscopy, Nano Lett. 15 (2015) 6996-7001. doi:10.1021/acs.nanolett.5b03008.

[24] A. Al-Afeef, W.P. Cockshott, I. MacLaren, S. McVitie, Electron tomography image reconstruction using data-driven adaptive compressed sensing, Scanning. 38 (2016) 251 276. doi:10.1002/sca.21271.

[25] B. Liu, H. Yu, S.S. Verbridge, L. Sun, G. Wang, Dictionary-learning-based reconstruction method for electron tomography, Scanning. 36 (2014) 377-383. doi:10.1002/sca.21127.

[26] E. Bladt, D.M. Pelt, S. Bals, K.J. Batenburg, Electron tomography based on highly limited data using a neural network reconstruction technique, Ultramicroscopy. 158 (2015) 81-88. doi:10.1016/j.ultramic.2015.07.001.

[27] K.E. MacArthur, T.J.A. Slater, S.J. Haigh, D. Ozkaya, P.D. Nellist, S. Lozano-Perez, Quantitative Energy-Dispersive X-Ray Analysis of Catalyst Nanoparticles Using a Partial Cross Section Approach, Microsc. Microanal. 22 (2016) 71-81. doi:10.1017/S1431927615015494.

[28] R.K. Leary, A. Kumar, P.J. Straney, S.M. Collins, S. Yazdi, R.E. Dunin-Borkowski, P.A. Midgley, J.E. Millstone, E. Ringe, Structural and Optical Properties of Discrete Dendritic Pt Nanoparticles on Colloidal Au Nanoprisms, J. Phys. Chem. C. (2016). http://pubs.acs.org/doi/abs/10.1021/acs.jpcc.6b02103 (accessed July 16, 2016).

[29] M.D. Guay, W. Czaja, M.A. Aronova, R.D. Leapman, Compressed Sensing Electron Tomography for Determining Biological Structure, Sci. Rep. 6 (2016) 27614. doi:10.1038/srep27614. 
[30] M. Pfannmöller, H. Heidari, L. Nanson, O.R. Lozman, M. Chrapa, T. Offermans, G. Nisato, S. Bals, Quantitative Tomography of Organic Photovoltaic Blends at the Nanoscale, Nano Lett. 15 (2015) 6634-6642. doi:10.1021/acs.nanolett.5b02437.

[31] T. Sanders, J.D. Roehling, K.J. Batenburg, B.C. Gates, A. Katz, P. Binev, I. Arslan, Advanced 3-D Reconstruction Algorithms for Electron Tomography, Microsc. Microanal. 20 (2014) 794-795. doi:10.1017/S1431927614005698.

[32] M.H. Gass, K.K.K. Koziol, A.H. Windle, P.A. Midgley, Four-Dimensional Spectral Tomography of Carbonaceous Nanocomposites, Nano Lett. 6 (2006) 376-379. doi:10.1021/nl052120g.

[33] A. Yurtsever, M. Weyland, D.A. Muller, Three-dimensional imaging of nonspherical silicon nanoparticles embedded in silicon oxide by plasmon tomography, Appl. Phys. Lett. 89 (2006) 151920. doi:10.1063/1.2360906.

[34] B. Goris, S. Bals, W. Van den Broek, J. Verbeeck, G. Van Tendeloo, Exploring different inelastic projection mechanisms for electron tomography, Ultramicroscopy. 111 (2011) 1262-1267. doi:10.1016/j.ultramic.2011.02.007.

[35] G. Haberfehlner, P. Bayle-Guillemaud, G. Audoit, D. Lafond, P.H. Morel, V. Jousseaume, T. Ernst, P. Bleuet, Four-dimensional spectral low-loss energy-filtered transmission electron tomography of silicon nanowire-based capacitors, Appl. Phys. Lett. 101 (2012) 063108. doi:10.1063/1.4742932.

[36] R.F. Egerton, Electron Energy-Loss Spectroscopy in the Electron Microscope, 3rd ed. 2011, Springer, New York, 2011.

[37] A. Hörl, A. Trügler, U. Hohenester, Tomography of Particle Plasmon Fields from Electron Energy Loss Spectroscopy, Phys. Rev. Lett. 111 (2013) 076801. doi:10.1103/PhysRevLett.111.076801.

[38] A. Hörl, A. Trügler, U. Hohenester, Full Three-Dimensonal Reconstruction of the Dyadic Green Tensor from Electron Energy Loss Spectroscopy of Plasmonic Nanoparticles, ACS Photonics. 2 (2015) 1429-1435. doi:10.1021/acsphotonics.5b00256.

[39] A. Losquin, M. Kociak, Link between Cathodoluminescence and Electron Energy Loss Spectroscopy and the Radiative and Full Electromagnetic Local Density of States, ACS Photonics. 2 (2015) 1619-1627. doi:10.1021/acsphotonics.5b00416.

[40] L.H.G. Tizei, Y.-C. Lin, M. Mukai, H. Sawada, A.-Y. Lu, L.-J. Li, K. Kimoto, K. Suenaga, Exciton Mapping at Subwavelength Scales in Two-Dimensional Materials, Phys. Rev. Lett. 114 (2015) 107601. doi:10.1103/PhysRevLett.114.107601.

[41] L.H.G. Tizei, Y.-C. Lin, A.-Y. Lu, L.-J. Li, K. Suenaga, Electron energy loss spectroscopy of excitons in two-dimensional-semiconductors as a function of temperature, Appl. Phys. Lett. 108 (2016) 163107. doi:10.1063/1.4947058.

[42] O.L. Krivanek, T.C. Lovejoy, N. Dellby, T. Aoki, R.W. Carpenter, P. Rez, E. Soignard, J. Zhu, P.E. Batson, M.J. Lagos, R.F. Egerton, P.A. Crozier, Vibrational spectroscopy in the electron microscope, Nature. 514 (2014) 209-212. doi:10.1038/nature13870.

[43] S. Meuret, L.H. Tizei, T. Auzelle, R. Songmuang, B. Daudin, B. Gayral, M. Kociak, Lifetime Measurements Well below the Optical Diffraction Limit, ACS Photonics. (2016). http://pubs.acs.org/doi/abs/10.1021/acsphotonics.6b00212 (accessed July 18, 2016).

[44] S. Meuret, L.H.G. Tizei, T. Cazimajou, R. Bourrellier, H.C. Chang, F. Treussart, M. Kociak, Photon Bunching in Cathodoluminescence, Phys. Rev. Lett. 114 (2015) 197401. doi:10.1103/PhysRevLett.114.197401. 
[45] A.A. Herzing, L.J. Richter, I.M. Anderson, 3D nanoscale characterization of thin-film organic photovoltaic device structures via spectroscopic contrast in the TEM 1, J. Phys. Chem. C. 114 (2010) 17501-17508.

[46] G. Möbus, R.C. Doole, B.J. Inkson, Spectroscopic electron tomography, Ultramicroscopy. 96 (2003) 433-451. doi:10.1016/S0304-3991(03)00106-2.

[47] T.J.A. Slater, A. Janssen, P.H.C. Camargo, M.G. Burke, N.J. Zaluzec, S.J. Haigh, STEMEDX tomography of bimetallic nanoparticles: A methodological investigation, Ultramicroscopy. 162 (2016) 61-73. doi:10.1016/j.ultramic.2015.10.007.

[48] C.S. Yeoh, D. Rossouw, Z. Saghi, P. Burdet, R.K. Leary, P.A. Midgley, The dark side of EDX tomography: modeling detector shadowing to aid 3D elemental signal analysis, Microsc. Microanal. 21 (2015) 759-764.

[49] D. Zanaga, T. Altantzis, L. Polavarapu, L.M. Liz-Marzán, B. Freitag, S. Bals, A New Method for Quantitative XEDS Tomography of Complex Heteronanostructures, Part. Part. Syst. Charact. 33 (2016) 396-403. doi:10.1002/ppsc.201600021.

[50] T. Slater, Y. Chen, G. Auton, N. Zaluzec, S. Haigh, X-Ray Absorption Correction for Quantitative Scanning Transmission Electron Microscopic Energy-Dispersive X-Ray Spectroscopy of Spherical Nanoparticles, Microsc. Microanal. 22 (2016) 440-447. doi:10.1017/S1431927616000064.

[51] D. Zanaga, T. Altantzis, J. Sanctorum, B. Freitag, S. Bals, An alternative approach for $\zeta$ factor measurement using pure element nanoparticles, Ultramicroscopy. 164 (2016) 11-16. doi:10.1016/j.ultramic.2016.03.002.

[52] P. Rueda-Fonseca, E. Robin, E. Bellet-Amalric, M. Lopez-Haro, M. Den Hertog, Y. Genuist, R. André, A. Artioli, S. Tatarenko, D. Ferrand, J. Cibert, Quantitative Reconstructions of 3D Chemical Nanostructures in Nanowires, Nano Lett. 16 (2016) 16371642. doi:10.1021/acs.nanolett.5b04489.

[53] D. Rossouw, P. Burdet, F. de la Peña, C. Ducati, B.R. Knappett, A.E. Wheatley, P.A. Midgley, Multicomponent signal unmixing from nanoheterostructures: Overcoming the traditional challenges of nanoscale $\mathrm{x}$-ray analysis via machine learning, Nano Lett. 15 (2015) 2716-2720.

[54] W. van Aarle, W.J. Palenstijn, J. De Beenhouwer, T. Altantzis, S. Bals, K.J. Batenburg, J. Sijbers, The ASTRA Toolbox: A platform for advanced algorithm development in electron tomography, Ultramicroscopy. 157 (2015) 35-47. doi:10.1016/j.ultramic.2015.05.002.

[55] F. de la Peña, J. Caron, L.F. Zagonel, P. Burdet, E. Prestat, iygr, G. Donval, V.T. Fauske, K.E. MacArthur, M. Nord, P. Jokubauskas, A. Garmannslund, J. Taillon, D. Johnstone, A. Eljarrat, T. Furnival, T. Ostasevicius, S. Mazzucco, M. Walls, M. Sarahan, B. Martineau, C. Gohlke, T. Aarholt, hyperspy: HyperSpy 1.0.1, 2016. http://zenodo.org/record/58841 (accessed August 11, 2016). 